\title{
Self-consistent approach to x-ray reflection from rough surfaces
}

\author{
I. D. Feranchuk, ${ }^{1}$ S. I. Feranchuk, ${ }^{1}$ and A. P. Ulyanenkove,* \\ ${ }^{1}$ Department of Theoretical Physics, Belarusian State University, 4 Fr. Skariny Avenue, 220080 Minsk, Republic of Belarus \\ ${ }^{2}$ Bruker AXS, Östl.Rheinbrückenstrasse 49, 76187 Karlsruhe, Germany
}

(Received 2 August 2006; revised manuscript received 17 November 2006; published 8 February 2007)

\begin{abstract}
A self-consistent analytical approach for specular x-ray reflection from interfaces with transition layers [I. D. Feranchuk et al., Phys. Rev. B 67, 235417 (2003)] based on the distorted-wave Born approximation (DWBA) is used for the description of coherent and incoherent $\mathrm{x}$-ray scattering from rough surfaces and interfaces. This approach takes into account the transformation of the modeling transition layer profile at the interface, which is caused by roughness correlations. The reflection coefficients for each DWBA order are directly calculated without phenomenological assumptions on their exponential decay at large scattering angles. Various regions of scattering angles are discussed, which show qualitatively different dependence of the reflection coefficient on the scattering angle. The experimental data are analyzed using the method developed.
\end{abstract}

DOI: 10.1103/PhysRevB.75.085414

PACS number(s): 61.10.Kw, 68.35.Ct, 61.10.Eq

\section{INTRODUCTION}

A theoretical description of specular and off-specular (diffuse) $x$-ray scattering from rough surfaces and interfaces is of special importance for the analysis of modern semiconductor and other nanoscale devices. As has been demonstrated in pioneering work, ${ }^{1}$ the imperfection of surfaces and interfaces is not comprehensively described by only the root mean square of the roughness amplitude $\sigma$; the roughness correlation length $L_{c}$ and fractal dimension $3-h$ have to be considered, too.

The most effective method for the calculation of x-ray scattering from rough surfaces has proved to be the distortedwave Born approximation (DWBA), which is extensively used in the literature (see, for example, Ref. 2). For the zeroth approximation of the DWBA, either the specular reflection from perfect plane interface (Fresnel reflection) or the reflection from interface modeled by transition layers with profile delivering an exact solution ${ }^{3}$ are usually used. The roughness correlations are taken into account in the firstorder DWBA on the deviation of a real scattering surface from the model one. However, diffuse scattering leads to a reduction of the intensity of specular (coherent) reflection due to conservation of radiation flux. To calculate this loss, typically the semiphenomenological Debye-Waller $F_{D W}$ $=\exp \left(-2 k_{z}^{2} \sigma^{2}\right)$ or Nevot-Croce ${ }^{4} F_{N C}=\exp \left(-2 k_{z} k_{1 z} \sigma^{2}\right)$ factor is used, which, however, depends not on the roughness correlations but solely on the parameter $\sigma$. Here $k_{z}=k \sin \alpha, k$ $=2 \pi / \lambda$ and $k_{1 z}=k \sqrt{\sin ^{2} \alpha+\chi}$ are the projections of $\mathrm{x}$-ray wave vectors onto the $z$ axis in a vacuum and medium, respectively; $\alpha$ is the incidence angle; $\chi(\lambda)<0$ is the $\mathrm{x}$-ray polarizability of medium.

However, the reflection coefficient from rough surfaces, as shown in Ref. 5, depends also on the roughness correlations. This dependence is described by the second-order DWBA, and both $F_{D W}$ and $F_{N C}$ are derived as limiting cases for the magnitude of roughness correlation. The renormalization of the coefficient $R_{s}(\alpha)$ in Ref. 5 has been performed with the accuracy of the second-order DWBA on the imperfection of the surface. As a result, the specular reflection coefficient depends both on the parameter $\sigma$ and on the roughness correlation. For large scattering angles, the phenomenological "exponentiating" operation was applied to $R_{s}(\alpha)$, which calculated the first and the second DWBA approximations by expanding the exponent into series. In real experiments, however, the exponential behavior of $R_{s}(\alpha)$ at large angles has not been observed. ${ }^{6}$ For example, for largeamplitude yet long wavy (long correlation length $L_{c}$ ) roughness, the X-ray scattering is close to the one from perfect surface, where $R_{s}(\alpha)$ decays as $R_{s}(\alpha) \sim \alpha^{-2}$ with an increase of the scattering angle. Moreover, the above assumption is not self-consistent: any renormalization of the Fresnel reflection coefficient is equivalent to the introduction of a transition layer at the interface, ${ }^{3}$ whereas solutions for perfect interfaces are used for the calculation of the corrections for the reflection coefficient and diffuse scattering intensity. The semiphenomenological description of the exponential behavior of the reflection coefficient at large scattering angles $\alpha$ has been recently done in Ref. 6 too.

The main goal of the present work is development of the method for calculation of specularly reflected intensity from rough surfaces and interfaces without any phenomenological assumptions. Recently, the solution of the x-ray scattering problem from transition layers with an arbitrary profile $\Lambda(z)$ has been reported, ${ }^{7}$ which delivers an analytical expression for the reflection and transmission coefficients from the profile $\Lambda(z)$ with a high accuracy. This self-consistent approach (SCA) formulates a consistent description of specular and diffuse scattering for the high-order DWBA. Thus, in the zeroth DWBA it is not necessary to fix a profile of the transition layer. The solution of the wave equation ${ }^{7}$ is used instead, where the variable profile $\Lambda\left(z, k_{z}\right)$ depends on $k_{z}$. Further, using a zeroth-order wave field, the cross section of $\mathrm{x}$-ray scattering averaged over the statistical distribution of roughness is calculated. The equation for the determination of the initial profile $\Lambda\left(z, k_{z}\right)$ follows from the zero condition for the sum of corrections to the specular reflection, which are caused by roughness correlations. The derived profile of transition layers as well as the renormalized reflection coefficient depend then on both $\sigma$ and $L_{c}$. If a more accurate calculation is required, this iteration scheme can be continued by tuning a profile in the way that the higher corrections 
to the reflection coefficient are set to zero. This selfconsistency of the zeroth potential is equivalent to partial summation of infinite DWBA series. A similar approach has been used for the solution of the wave equation for discrete spectrum..$^{8}$

The nonperturbative description of $\mathrm{x}$-ray scattering from rough surfaces has been recently considered in Refs. 9 and 10. In the former publication, the wave equation has been solved by a method different than DWBA, whereas in the latter article the integral equation for the angular distribution of scattered radiation has been obtained, which is valid for any values of roughness and correlation length. However, both approaches result in cumbersome expressions for the scattered intensity and are not easy to use for the fitting of real experimental measurements. In Refs. 11 and 12, the variational profile parameters have been also used for the description of diffuse scattering. Contrary to the approach presented here, the authors used a fixed-exponential form for renormalization of the reflection and transmission coefficients. Caticha ${ }^{13}$ has proposed a self-consistent method for specular x-ray reflectivity from an arbitrary profile. A detailed comparison of his approach with the one developed by us was done in Ref. 7.

The article is structured in the following way. In Sec. II, the general DWBA scheme is discussed for wave equations, which describes the transmission of $\mathrm{x}$ rays through the boundary between two media modeled by transition layers with an arbitrary profile. In Sec. III, a self-consistent method for the calculation of the intensity of specular reflection is presented taking into consideration the second-order DWBA and wave fields derived in Ref. 7. In Sec. IV, the experimental data are evaluated by the method developed and characteristic intervals for scattering angles are distinguished, where the reflected intensity depends on the scattering angle $\alpha$ in different ways.

\section{DWBA IN THE CASE OF A TRANSITION LAYER WITH ARBITRARY PROFILE}

The dielectric constant of any medium for $\mathrm{x}$ rays is a scalar value, making the polarization effects negligible, and thus the propagation of electromagnetic waves with arbitrary polarization is described by the solution $\phi_{\vec{k}}(\vec{r})$ of the scalar wave equation ${ }^{2}$

$$
\left[\Delta+k^{2}-V(\vec{r})\right] \phi_{\vec{k}}(\vec{r})=0,
$$

with standard asymptotical boundary conditions, which correspond to scattering on the surface that is unlimited in the direction $x$ (Ref. 3):

$$
\begin{gathered}
\phi_{\vec{k}}(\vec{r}) \sim e^{i \vec{k} \cdot \vec{r}}+R(\vec{k}, \vec{p}) e^{i \vec{p} \cdot \vec{r}}, \quad p_{z}<0, \quad z \rightarrow-\infty, \\
\phi_{\vec{k}}(\vec{r}) \sim T(\vec{k}, \vec{p}) e^{i \vec{p} \cdot \vec{r}}, \quad p_{z}>0, \quad z \rightarrow \infty,
\end{gathered}
$$

where $\vec{k}$ is the wave vector of incident and $\vec{p}$ of scattered $\left(p^{2}=k^{2}\right)$ waves, respectively, $R(\vec{k}, \vec{p})$ and $T(\vec{k}, \vec{p})$ are the amplitudes of the reflected and transmitted waves, the condition $\vec{p}_{\perp}=\vec{k}_{\perp}$ defines the case of specular reflection, and $\vec{p}_{\perp} \neq \vec{k}_{\perp}$ is the case of diffuse scattering. ${ }^{1}$

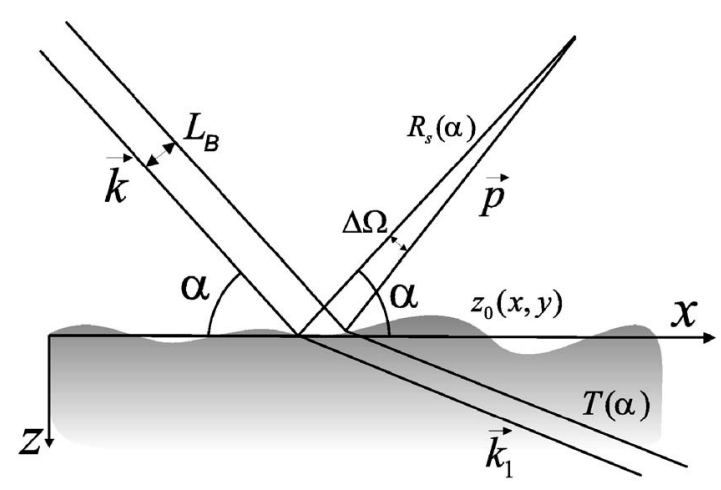

FIG. 1. Scattering of $\mathrm{x}$ rays from a rough surface. The reflection $R(\alpha)$ and transmission $T(\alpha)$ coefficients depend on the scattering angle $\alpha$ and are expressed through the scattering amplitude $T(\vec{k}, \vec{p})$ for different wave vectors of the final state (2).

The angle $\alpha \sim \alpha_{c}=\sqrt{|\chi|} \ll 1$, corresponding to effective interaction length $l \sim \lambda \alpha^{-1}$, plays an essential role in the scattering from surface. Because the value of $l$ exceeds essentially the atomic dimension, the interaction potential between $x$ rays and semi-infinite media is determined from the expression $^{3}$

$$
V(\vec{r})=k^{2} \chi(\lambda) H\left[z-z_{0}(x, y)\right],
$$

where $H\left[z-z_{0}(x, y)\right]$ is the Heaviside function with random function argument $z_{0}(x, y)$ defining the surface roughness (Fig. 1). The ideal, perfectly smooth surface corresponds to $z_{0}=0$.

The main problem in the solving of Eq. (1) is that the function $\phi_{\vec{k}}(\vec{r})$ is a complex nonlinear functional of random function $z_{0}(x, y)$. This fact does not permit us to construct a closed equation for the function $\left\langle\phi_{\vec{k}}(\vec{r})\right\rangle$ averaged over the distribution $z_{0}(x, y)$. The common solution for this problem in x-ray reflectometry is based on the proportionality of the amplitude of specular (coherent) reflection to the entire sample surface $\sim S$. At the same time, the amplitude of diffuse (incoherent) scattering is proportional to $\sim \sqrt{S}$ and is small even for large roughness, which makes it possible to take it into account by the DWBA. ${ }^{3}$ The potential (3) is written in the form

$$
\begin{gathered}
V(\vec{r})=V_{0}(z)+\xi V_{1}(\vec{r}) \\
\equiv V_{0}(z)+\xi\left\{k^{2} \chi(\lambda) H\left[z-z_{0}(x, y)\right]-V_{0}(z)\right\}, \\
V_{0}(z) \equiv k^{2} \chi(\lambda) \Lambda(z),
\end{gathered}
$$

where $V_{0}(z)$ is the one-dimensional coherent potential of the transition layer, taking into account the influence of roughness on specular reflectivity; the dimensionless function $\Lambda(z)$ defines the profile of this layer, ${ }^{3}$ and by definition $\Lambda(z) \rightarrow 0$, $z \rightarrow-\infty, \Lambda(z) \rightarrow 1$, and $z \rightarrow \infty$. The formal parameter $\xi$ is introduced to order the terms of the perturbation series on their smallness, and in the final expressions it will be set to zero 
$\xi=1$. Using the potential (4), Eq. (1) is transformed to the integral equation

$$
\phi_{\vec{k}}(\vec{r})=\phi_{\vec{k}}^{(0)}(\vec{r})+\xi \int d \vec{r}^{\prime} G_{k}\left(\vec{r}, \vec{r}^{\prime}\right) V_{1}\left(\vec{r}^{\prime}\right) \phi_{\vec{k}}\left(\vec{r}^{\prime}\right) .
$$

The zeroth approximation $\phi_{\vec{k}}^{(0)}(\vec{r})$ is derived from the equation with potential $V_{0}(z)$ :

$$
\left[\Delta+k^{2}-V_{0}(z)\right] \phi_{\vec{k}}^{(0)}(\vec{r})=0, \quad \phi_{\vec{k}}^{(0)}(\vec{r})=e^{i \vec{k}_{\perp} \cdot \vec{r}} \varphi_{k_{z}}(z),
$$

and boundary conditions for the one-dimensional wave equation are

$$
\begin{gathered}
{\left[\frac{d^{2}}{d z^{2}}+k_{z}^{2}-k^{2} \chi \Lambda(z)\right] \varphi_{k_{z}}(z)=0, \quad k_{z}=\sqrt{k^{2}-k_{\perp}^{2}},} \\
\varphi_{k_{z}}(z) \sim e^{i k_{z} z}+R^{(0)}\left(k_{z}\right) e^{-i k_{z} z}, \quad z \rightarrow-\infty, \\
\varphi_{k_{z}}(z) \sim T^{(0)}\left(k_{z}\right) e^{i k_{1} z}, \quad k_{1 z}=\sqrt{k_{z}^{2}+k^{2} \chi}, z \rightarrow \infty,
\end{gathered}
$$

which deliver the zeroth approximation $R^{(0)}\left(k_{z}\right)$ for the specular reflection coefficient. The Green function $G_{k}(\vec{r}, \vec{r})$ satisfies the equation

$$
\left\{\Delta+k^{2}-V_{0}(z)\right\} G_{k}\left(\vec{r}, \vec{r}^{\prime}\right)=\delta\left(\vec{r}-\vec{r}^{\prime}\right),
$$

and according to the general theory of differential equations can be expressed ${ }^{5}$ through two fundamental solutions of Eq. (7):

$$
\begin{gathered}
G_{k}\left(\vec{r}, \vec{r}^{\prime}\right)=\int_{\left|\vec{k}_{\perp}^{\prime}\right|<k} \frac{d \vec{k}_{\perp}^{\prime}}{4 \pi^{2}} e^{i \vec{k}_{\perp} \cdot\left(\vec{r}-\vec{r}^{\prime}\right)} g_{k_{z}^{\prime}}\left(z, z^{\prime}\right), \quad k_{z}^{\prime}=\sqrt{k^{2}-k_{\perp}^{\prime 2}}, \\
g_{k_{z}^{\prime}}\left(z, z^{\prime}\right)=-\frac{\varphi_{k_{z}^{\prime}}\left(z_{1}\right) \varphi_{-k_{z}^{\prime}}\left(z_{2}\right)}{W}, \quad z_{1}=\min \left(z, z^{\prime}\right), \\
z_{2}=\max \left(z, z^{\prime}\right), \\
W=\varphi_{-k_{z}^{\prime}} \frac{d \varphi_{k_{z}^{\prime}}}{d z}-\varphi_{k_{z}^{\prime}} \frac{d \varphi_{-k_{z}^{\prime}}}{d z} .
\end{gathered}
$$

Using Eqs. (6)-(9) and iteration scheme for approximate solution of Eq. (5), the formal series of DWBA's can be obtained for the scattering amplitude $T(\vec{k}, \vec{p})$. The square of this amplitude delivers in the differential cross section of $\mathrm{x}$-ray scattering in the half plane $z<0 .{ }^{5}$ The explicit expressions for the terms of this series up to the second order of the potential $V_{1}(\vec{r})$-i.e., the parameter $\xi$-are

$$
\begin{gathered}
T(\vec{k}, \vec{p}) \approx T^{(0)}(\vec{k}, \vec{p})+\xi T^{(1)}(\vec{k}, \vec{p})+\xi^{2} T^{(2)}(\vec{k}, \vec{p})+\cdots, \\
T^{(0)}(\vec{k}, \vec{p})=R^{(0)}\left(k_{z}\right)(2 \pi)^{2} \delta\left(\vec{p}_{\perp}-\vec{k}_{\perp}\right), \\
R^{(0)}\left(k_{z}\right)=\int d z e^{i k_{z} z} V_{0}(z) \varphi_{k_{z}}, \\
T^{(1)}(\vec{k}, \vec{p})=\int d \vec{r} e^{i\left(\vec{p}_{\perp}-\vec{k}_{\perp}\right) \cdot \vec{r}} \varphi_{-k_{z}}^{*} V_{1}(\vec{r}) \varphi_{p_{z}},
\end{gathered}
$$

$$
\begin{aligned}
T^{(2)}(\vec{k}, \vec{p})= & \int d \vec{r} d \vec{r}^{\prime} \int_{\left|\vec{k}_{\perp}^{\prime}\right|<k} \frac{d \vec{k}_{\perp}^{\prime}}{k_{z}^{\prime}} e^{i\left(\vec{k}_{\perp}^{\prime}-\vec{k}_{\perp}\right) \cdot \vec{r}} e^{i\left(\vec{p}_{\perp}-\vec{k}_{\perp}^{\prime}\right) \cdot \vec{r}^{\prime}} \varphi_{-k_{z}}^{*}(z) \\
& \times V_{1}\left(\vec{r}^{\prime}\right) \varphi_{k_{z}^{\prime}}(z) \varphi_{-k_{z}^{\prime}}^{*}\left(z^{\prime}\right) V_{1}\left(\vec{r}^{\prime}\right) \varphi_{p_{z}}\left(z^{\prime}\right)
\end{aligned}
$$

where $R^{(0)}\left(k_{z}\right)$ is the specular reflection coefficient from the interface with profile $\Lambda(z)$. The observed differential cross section of $\mathrm{x}$-ray scattering is calculated as ${ }^{1}$

$$
\frac{d \sigma}{d \Omega}=\frac{1}{16 \pi^{2}}\left\langle|T(\vec{k}, \vec{p})|^{2}\right\rangle,
$$

after averaging $\langle\cdots\rangle$ over the ensemble of random functions $z_{0}(x, y)$. In the zeroth approximation, the only specular reflection $\sim\left|R^{(0)}\left(k_{z}\right)\right|^{2}$ is obtained from Eq. (10). The diffuse scattering ${ }^{1}$ is caused by the fluctuations of the scattering square amplitude-i.e., appears in the first-order DWBA $\sim \xi^{2}\left(\left\langle\left|T^{(1)}(\vec{k}, \vec{p})\right|^{2}\right\rangle-\left|\left\langle T^{(1)}(\vec{k}, \vec{p})\right\rangle\right|^{2}\right)$. Thus, its contribution to the cross section has the same order as the term depending on the second-order DWBA $\sim \xi^{2} R^{(0)}\left(k_{z}\right)\left\langle T^{(2)}(\vec{k}, \vec{p})\right\rangle$, and therefore, for the calculation of the scattering cross section both contributions have to be taken into account. ${ }^{5}$ The validity conditions for the approach presented coincide rather with the validity area of the DWBA than the one of perturbation theory. Concerning the validity of DWBA convergence, it might be defined by the ratio of the spectral intensity of specular reflection to diffusely scattered intensity. ${ }^{3}$ This is because of the cross section of x-ray scattering from the averaged potential $V_{0}(z)$ is proportional to the square of the sample surface $S$, whereas scattering from fluctuations depends on the roughness rms, correlation length, and scattering angle.

To use formulas (6)-(11), the analytical expression for the profile of the zeroth approximation $\Lambda(z)$ has to be chosen and then the explicit expression for $\varphi_{k_{z}}(z)$ has to be found. In the meantime, a definite distribution model for the ensemble of random functions $z_{0}(x, y)$ has to be selected. In Ref. 5, the perfect plane interface has been selected as the zeroth approximation, which is a typical choice for DWBA applications:

$$
\begin{gathered}
\Lambda(z) \approx \Lambda_{0}(z)=H(z), \\
\varphi_{k_{z}}^{(0)}(z)=\left(e^{i k_{z} z}+r^{(0)} e^{-i k_{z} z}\right) H(-z)+t^{(0)} e^{i k_{1 z} z} H(z), \\
r^{(0)}\left(k_{z}\right)=\frac{k_{z}-k_{1 z}}{k_{1 z}+k_{z}}, \quad t^{(0)}\left(k_{z}\right)=\frac{2 k_{z}}{k_{1 z}+k_{z}},
\end{gathered}
$$

where $r^{(0)}$ and $t^{(0)}$ are expressions for the Fresnel reflection and transmission coefficients for an ideal surface. For this choice of initial profile, the first- and second-order DWBA corrections for the specular reflection coefficient, accounting roughness correlations, can be calculated. ${ }^{5}$ For small scattering angles, the specular reflection coefficient varies in dependence on the roughness correlations within the limits 


$$
r^{(0)}\left(k_{z}\right)\left(1-2 \sigma^{2} k_{z}^{2}\right)<R_{s}\left(k_{z}\right)<r^{(0)}\left(k_{z}\right)\left(1-2 \sigma^{2} k_{z} k_{1 z}\right) .
$$

At small scattering angles $k_{z} \sigma \ll 1$, the calculations of Ref. 5 are completely microscopical. They demonstrate that reconstruction of the transition layer, which influences the reflection coefficient, depends on both averaged roughness and roughness correlations. However, at larger scattering angles the formula for specular reflection, inequality (13), fails, and accounting for high-order terms in (10) is necessary, which is difficult to realize in practice. A commonly accepted approach to solve this problem is an additional phenomenological assumption about terms in parentheses in (13), which are supposed to be the first terms in the expansion over the parameter $k_{z} \sigma$ of the Gaussian exponent ("exponentiating" operation). The latter makes a renormalization of the reflection coefficient:

$$
r^{(0)}\left(k_{z}\right) e^{-2 \sigma^{2} k_{z}^{2}}<R_{s}\left(k_{z}\right)<r^{(0)}\left(k_{z}\right) e^{-2 \sigma^{2} k_{z} k_{1 z}} .
$$

Depending on the correlations, this exponent reduces to the Debye-Waller or Nevot-Croce exponent. ${ }^{5}$

This simple renormalization describes the experimental data well when the measured sample possesses a roughness of small amplitude. However, for larger $\sigma$ values, the observed $R_{S}\left(k_{z}\right)$ may considerably differ from the exponential one. Therefore, for large roughnesses an alternative phenomenological approximation has been proposed, ${ }^{6}$ which introduces an additional parameter, a maximal roughness amplitude. This approach has proved to describe well the experimental data from samples with a very rough surface, as shown in Ref. 6. Nevertheless, the solution of the following problem remains actual and demanded: is a microscopical description of the reflection from an imperfect surface possible for arbitrary scattering angles by a low-order DWBA and without any additional assumptions?

\section{SELF-CONSISTENT APPROACH TO THE CALCULATION OF X-RAY SCATTERING}

The physical reason for the necessity of the renormalization of the specular reflection coefficient is conservation of the photons flux. The emission of diffuse $\mathrm{x}$-ray scattering is compensated for by the reduction of the intensity of specular reflection. ${ }^{5}$ The calculated intensity of diffuse scattering depends on the DWBA order applied; therefore, the corrections for $R^{(0)}\left(k_{z}\right)$ also vary in each higher DWBA. These corrections, however, cannot be summed up in the general case, which causes the phenomenological modeling of $R^{(0)}\left(k_{z}\right)$ in conventional theories.

The key point of the proposed self-consistent approach is not to fix the profile of the transition layer initially, but to consider

$$
\Lambda^{(0)}(z) \Rightarrow \Lambda\left(k_{z}, z\right)
$$

as a variational function changing with the DWBA order and depending on $k_{z}$. This additional degree of freedom can be used for the transformation of the differential cross section of x-ray scattering, simulated on the basis of the DWBA. The physical meaning of the variational profile dependence on $k_{z}$ is conditioned by the dependence of the averaged within the higher DWBA order surface potential on the projection of the correlation length onto the incidence beam [see Eq. (42) below].

Assume that the differential cross section of x-ray scattering (11) is calculated with an accuracy up to the second order of $\xi$. Then performing an averaging over the ensemble of random functions $z_{0}(x, y)$, it is expressed as ${ }^{1}$

$$
\begin{gathered}
\frac{d \sigma}{d \Omega}=\frac{1}{16 \pi^{2}}|T(\vec{k}, \vec{p})|^{2}, \\
|T(\vec{k}, \vec{p})|^{2}= \\
\left\{\left|T^{(0)}\left(\left\{\Lambda\left(k_{z}, z\right)\right\}\right)+\xi\left\langle T^{(1)}\left(\left\{\Lambda\left(k_{z}, z\right)\right)\right\}\right\rangle\right|^{2}\right. \\
\left.+2 \xi^{2} \operatorname{Re}\left[T^{(0) *}\left(\left\{\Lambda\left(k_{z}, z\right)\right\}\right)\left\langle T^{(2)}\left(\left\{\Lambda\left(k_{z}, z\right)\right\}\right)\right\rangle\right]\right\} \\
+\xi^{2}\left[\left\langle\left|T^{(1)}(\vec{k}, \vec{p})\right|^{2}\right\rangle-\left|\left\langle T^{(1)}(\vec{k}, \vec{p})\right\rangle\right|^{2}\right] .
\end{gathered}
$$

All the terms in this expression are functionals of the profile $\Lambda\left(k_{z}, z\right)$. This profile can be chosen in such a way that the sum of all terms in specular reflection $\left(\vec{p}_{\perp}=\vec{k}_{\perp}\right)$, except the first one, equals zero:

$$
\begin{aligned}
& \left\{\xi^{2}\left\{\left|\left\langle T^{(1)}\left(\left\{\Lambda\left(k_{z}, z\right)\right)\right\}\right\rangle\right|^{2}+\left[\left\langle\left|T^{(1)}(\vec{k}, \vec{p})\right|^{2}\right\rangle-\left|\left\langle T^{(1)}(\vec{k}, \vec{p})\right\rangle\right|^{2}\right]\right\}\right. \\
& \quad+2 \operatorname{Re}\left\{T ^ { ( 0 ) * } ( \{ \Lambda ( k _ { z } , z ) \} ) \left[\xi\left\langle T^{(1)}\left(\left\{\Lambda\left(k_{z}, z\right)\right\}\right)\right\rangle\right.\right. \\
& \left.\left.\left.\quad+\xi^{2}\left\langle T^{(2)}\left(\left\{\Lambda\left(k_{z}, z\right)\right\}\right)\right\rangle\right]\right\}\right\}_{\left(\vec{p}_{\perp}=\vec{k}_{\perp}\right)}=0 .
\end{aligned}
$$

The solution to this functional equation delivers the function $\Lambda\left(k_{z}, z\right)$, which is a profile of the transition layer, and the specular reflection coefficient is

$$
R\left(k_{z}\right) \approx T^{(0)}\left(k_{z},\left\{\Lambda\left(k_{z}, z\right)\right\}\right),
$$

with already performed renormalization. Thus, the scattering cross section for all exit angles is separated into coherent specular and incoherent diffuse scattering.

The principal problem of algorithm realized in Eqs. (15)-(17) is the analytical solution of the equation for the zeroth approximation (7) for arbitrary profile $\Lambda(z)$. This solution has been proposed in Ref. 7 as an ansatz:

$$
\varphi_{k_{z}}(z)=\Lambda(z) T^{(0)} e^{i k_{1} z}+[1-\Lambda(z)]\left(R_{s}^{(0)} e^{-i k_{z} z}+e^{i k_{z} z}\right) .
$$

The reflection and transmission coefficients in Eq. (19), are expressed through the potential amplitude in Eq. (3), A $=k^{2} \chi(\lambda)$, and integrals of function $\Lambda(z)$ :

$$
R_{s}^{(0)}\left(k_{z}\right)=\frac{i A\left[K\left(2 k_{z}\right) L\left(k_{1 z}-k_{z}\right)\left(k_{1 z}+k_{z}\right)-K(0) L\left(k_{1 z}+k_{z}\right)\left(k_{1 z}-k_{z}\right)\right]-2 k_{z} L\left(k_{1 z}+k_{z}\right)\left(k_{1 z}-k_{z}\right)}{i A\left[K\left(-2 k_{z}\right) L\left(k_{1 z}+k_{z}\right)\left(k_{1 z}-k_{z}\right)-K(0) L\left(k_{1 z}-k_{z}\right)\left(k_{1 z}+k_{z}\right)\right]+2 k_{z} L\left(k_{1 z}-k_{z}\right)\left(k_{1 z}+k_{z}\right)},
$$




$$
\begin{gathered}
T^{(0)}\left(k_{z}\right)=\frac{A^{2}\left[K^{2}(0)-K\left(2 k_{z}\right) K\left(-2 k_{z}\right)\right]+4 k_{z}^{2}}{2 i A\left[K\left(-2 k_{z}\right) L\left(k_{1 z}+k_{z}\right)\left(k_{1 z}-k_{z}\right)-K(0) L\left(k_{1 z}-k_{z}\right)\left(k_{1 z}+k_{z}\right)\right]+4 k_{z} L\left(k_{1 z}-k_{z}\right)\left(k_{1 z}+k_{z}\right)}, \\
K(r)=\int_{-\infty}^{\infty} d z e^{i r z} \Lambda(z)[1-\Lambda(z)], \quad L(r)=\int_{-\infty}^{\infty} d z e^{i r z} \Lambda(z) \Lambda^{\prime}(z) .
\end{gathered}
$$

As shown in Ref. 7, the function (19) with coefficients (20) and (21) approximates ${ }^{7}$ the solution for Eq. (3) with high accuracy for different profiles of the transition layer. Both amplitudes and phases of the transmission and reflection coefficients are correctly calculated within the entire angular region already in zeroth approximation. Moreover, the iteration scheme for exact solution is established and converges quickly to exact solution.

For the first DWBA order, Eq. (17) with perturbation potential (4) is

$$
\begin{aligned}
\left\langle T^{(1)}\left(\left\{\Lambda^{(0)}(z)\right\}\right)\right\rangle= & \int d \vec{r} e^{i\left(\vec{p}_{\perp}-\vec{k}_{\perp}\right) \cdot \vec{r}} \varphi_{-k_{z}}^{*}(z)\left[\left\langle H\left[z-z_{0}(x, y)\right]\right\rangle\right. \\
& \left.-\Lambda^{(0)}(z)\right] \varphi_{k_{z}}(z)=0
\end{aligned}
$$

and the solution for the profile $\Lambda^{(0)}(z)$ is

$$
\Lambda^{(0)}(z)=\left\langle H\left[z-z_{0}(x, y)\right]\right\rangle .
$$

Thus, in the first-DWBA-order SCA results in a known fact: the specular reflection from rough surfaces depends on the potential of the transition layer, which is the result of the averaging of a real potential over the surface. Assuming the Gaussian distribution of roughness amplitudes on the surface, the profile of the transition layer is expressed through the error function ${ }^{4}$

$$
\begin{aligned}
\Lambda^{(0)}(z) & =\left\langle H\left[z-z_{0}(x, y)\right]\right\rangle=\frac{1}{\sigma \sqrt{2 \pi}} \int_{-\infty}^{z} d z_{0} e^{-z_{0}^{2} / 2 \sigma^{2}} \\
& =\Phi\left(\frac{z}{\sigma \sqrt{2}}\right) .
\end{aligned}
$$

Figure 2 shows the simulation (dashed line) of specular x-ray reflectivity of wavelength $\lambda=1.54055 \AA$ from crystal $\mathrm{Si}_{0.65} \mathrm{Ge}_{0.35}\left(\chi=-1.99 \times 10^{-5}+i 5.27 \times 10^{-7}\right)$ with transition layer of profile (23) with $\sigma=40 \AA$ made by formula (20). For comparison, solution of the wave equation (7) for this profile based on Parratt's formalism ${ }^{14}$ (solid line) and reflectivity obtained by renormalization of the Fresnel coefficient by $F_{N C}$ (dots) are shown, too. Because of the profile's natural edge, the numerical solution of (7) does not coincide with the exact result at large scattering angles, which is given by the Born approximation and is an exponential asymptotic of the reflection coefficient. ${ }^{3}$ At the same time, the interpolation by $F_{N C}$ and self-consistent approximation both result in an exact asymptotic formula. ${ }^{7}$
For the second-order DWBA, the correlation of roughness ${ }^{1}$ has to be taken into account in the averaging of Eq. (16). For homogeneous surfaces and the standard definition of the correlation function,

$$
\left\langle\left[z_{0}\left(\vec{r}_{\perp}\right)-z_{0}\left(\vec{r}_{\perp}+\vec{R}_{\perp}\right)\right]^{2}\right\rangle=g\left(R_{\perp}\right),
$$

with fractal model ${ }^{1}$ used here and further:

$$
g\left(R_{\perp}\right)=2 \sigma^{2}\left(1-e^{-\left(R_{\perp} / L_{c}\right)^{2 h}}\right),
$$

where $h$ is the fractal dimension and $L_{c}$ is the correlation length.

The x-ray scattering cross section from potential (3) with accuracy up to $\xi^{2}$ is obtained by averaging of Eq. (16) on a Gaussian distribution of the random function $z_{0}(x, y)$ with the correlator (25):

$$
\frac{d \sigma}{d \Omega}=\frac{k^{4}|\chi|^{2}}{4} S|T(\vec{k}, \vec{p})|^{2},
$$

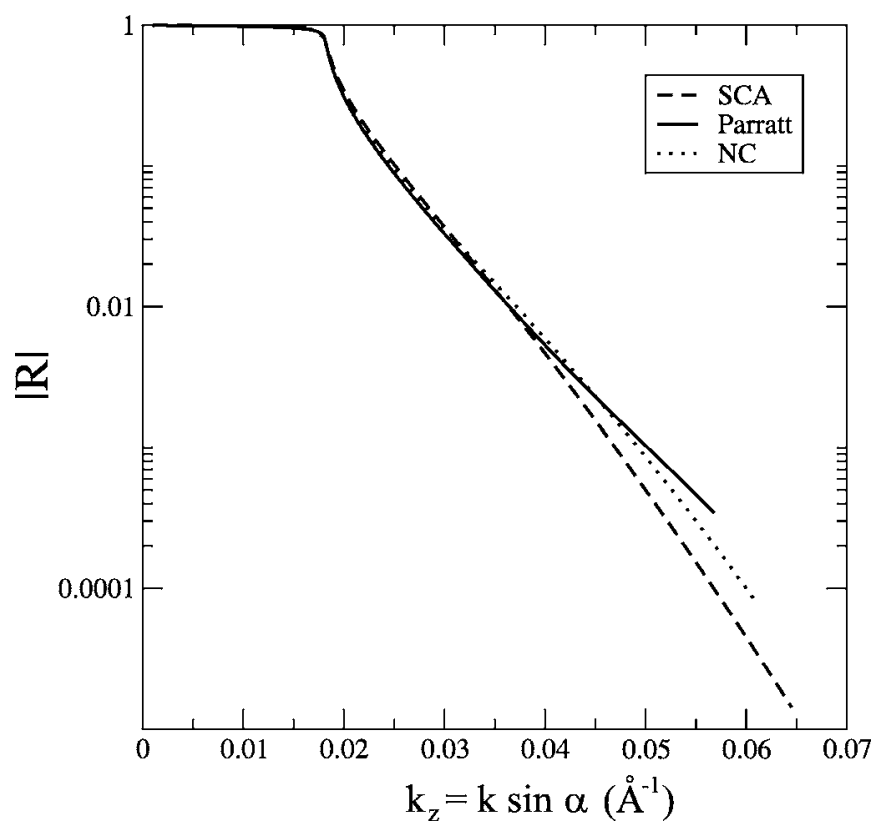

FIG. 2. The simulated $\mathrm{x}$-ray reflectivity curves from a rough $\mathrm{Si}_{0.65} \mathrm{Ge}_{0.35}$ layer on a $\mathrm{Si}$ substrate by different methods: numerical solution of Parratt's equation for the erf profile (solid line), renormalized by the Nevot-Croce factor the reflection coefficient (dots), and SCA in the first DWBA order (dashed line) 


$$
\begin{aligned}
|T(\vec{k}, \vec{p})|^{2}= & \delta\left(\vec{p}_{\perp}-\vec{k}_{\perp}\right)\left\{\left|T^{(0)}\left(k_{z},\left\{\Lambda^{(1)}(z)\right\}\right)+\xi B\left(k_{z}\right)\right|^{2}\right. \\
& \left.+2 \xi^{2} k^{2} \operatorname{Re}\left[T^{(0)^{*}}\left(k_{z},\left\{\Lambda^{(1)}(z)\right\}\right) \chi T^{(2)}\left(k_{z}\right)\right]\right\} \\
& +\xi^{2} K(\vec{k}, \vec{p}),
\end{aligned}
$$

where $S$ is the surface area of the sample. All the terms in (27) have a clear physical interpretation and are calculated as described below. The function

$$
T^{(0)}\left(k_{z},\left\{\Lambda^{(1)}(z)\right\}\right)=\int_{-\infty}^{\infty} d z e^{i k_{z} z} \Lambda^{(1)}(z) \varphi_{k_{z}}(z)
$$

is the amplitude of specular reflection from the profile $\Lambda^{(1)}(z)$. The additional contribution of the first order $B\left(k_{z}\right)$ into the specular amplitude is caused by the difference be- tween the function $\Lambda^{(1)}(z)$ and profile (24), which is taken as the error function $\Lambda^{(0)}(z)$. The latter does not depend, by definition, on the roughness correlation:

$$
B\left(k_{z}\right)=\int_{-\infty}^{\infty} d z \varphi_{-k_{z}}^{*}(z)\left[\Lambda^{(0)}(z)-\Lambda^{(1)}(z)\right] \varphi_{k_{z}}(z) .
$$

The term $T^{(2)}\left(k_{z}\right)$ in (27) corresponds to a double scattering of the wave field within the media ${ }^{5}$ and is calculated as the second-order correction in the solution of Eq. (5). The term $K(\vec{k}, \vec{p})$ is a single incoherent scattering depending on the root-mean-square fluctuation $\left[\left\langle V^{2}\right\rangle-\langle V\rangle^{2}\right]$ of the scattering potential (3) after averaging over the correlated roughness distribution:

$$
\begin{aligned}
& T^{(2)}(\vec{k})= \int_{\left|\vec{k}_{\perp}^{\prime}\right|<k} \frac{d \vec{k}_{\perp}^{\prime}}{k_{z}^{\prime}} \int_{-\infty}^{\infty} d z \int_{-\infty}^{\infty} d z^{\prime} \int d \vec{R}_{\perp} e^{i\left(\vec{k}_{\perp}^{\prime}-\vec{k}_{\perp}\right) \cdot \vec{R}_{\perp}} \varphi_{-k_{z}}^{*}\left(z^{\prime}\right) \varphi_{k_{z}^{\prime}}\left(z^{\prime}\right) \varphi_{-k_{z}^{\prime}}^{*}(z) \varphi_{k_{z}}(z) \\
& \times\left\{\int_{-\infty}^{\infty} d a_{1} \int_{-\infty}^{\infty} d a_{2} W\left(a_{1}, a_{2}, R_{\perp}\right) H\left[z^{\prime}-a_{1}\right] H\left[z-a_{2}\right]+\left[\Lambda^{(0)}(z)-\Lambda^{(1)}(z)\right]\left[\Lambda^{(0)}\left(z^{\prime}\right)-\Lambda^{(1)}\left(z^{\prime}\right)\right]\right\}, \\
& K(\vec{k}, \vec{p})=\frac{1}{4 \pi^{2}} \int_{-\infty}^{\infty} d z \int_{-\infty}^{\infty} d z^{\prime} \int d \vec{R}_{\perp} \int_{-\infty}^{\infty} d a_{1} \int_{-\infty}^{\infty} d a_{2} W\left(a_{1}, a_{2}, R_{\perp}\right) e^{i\left(\vec{p}_{\perp}-\vec{k}_{\perp}\right) \cdot \vec{R}_{\perp}} \varphi_{-p_{z}}^{*}\left(z^{\prime}\right) H\left[z^{\prime}-a_{1}\right] \varphi_{k_{z}}^{*}\left(z^{\prime}\right) \varphi_{-p_{z}}(z) H\left[z-a_{2}\right] \varphi_{k_{z}}(z) .
\end{aligned}
$$

The functions $\varphi_{k_{z}}(z)$ are the solutions of the wave equation (7) with profile $\Lambda^{(1)}(z)$, and the two-dimensional distribution of the roughness amplitudes $W\left(a_{1}, a_{2}, R_{\perp}\right)$ is

$$
\begin{aligned}
W\left(a_{1}, a_{2}, R_{\perp}\right)= & \frac{1}{\pi \sigma}\left[\frac{1}{\sqrt{2 g\left(R_{\perp}\right)}} e^{-\left(a_{1}+a_{2}\right)^{2} / 4 \sigma^{2}} e^{-\left(a_{1}-a_{2}\right)^{2} / 2 g\left(R_{\perp}\right)}\right. \\
& \left.-\frac{1}{2 \sigma} e^{-\left(a_{1}^{2}+a_{2}^{2}\right) / 2 \sigma^{2}}\right]
\end{aligned}
$$

with the correlation function $g\left(R_{\perp}\right)$ from Eq. (26).

\section{CALCULATION OF X-RAY REFLECTIVITY}

The differential cross section (27) describes both specular $\left(\vec{p}_{\perp}=\vec{k}_{\perp}\right)$ and diffuse scattering $K(\vec{k}, \vec{p})$, when the transverse component of the wave vector is not preserved. The term $K(\vec{k}, \vec{p})$ is nonzero for $\vec{p}_{\perp}=\vec{k}_{\perp}$, and the variational profile $\Lambda^{(1)}(z)$ has to be calculated taking in consideration this term in Eq. (17). To proceed with the simulations, the number of quanta, $N(\vec{n})$, registered by the detector in the direction of the unit vector $\vec{n}=\left(\vec{k}_{\perp} / k,-k_{z} / k\right)$, which corresponds to specular reflection, is calculated. The value of $N(\vec{n})$ depends on the width of angular cone $\Delta \Omega$ covered by detector (Fig. 1):

$$
N(\vec{n})=I_{0} \int d \vec{n}^{\prime} f\left(\vec{n}-\vec{n}^{\prime}\right) \frac{d \sigma}{d \Omega^{\prime}},
$$

$$
f\left(\vec{n}-\vec{n}^{\prime}\right)=\exp \left(-\frac{\Delta_{x}^{2}}{\delta_{x}^{2}}-\frac{\Delta_{y}^{2}}{\delta_{y}^{2}}\right), \quad \vec{\Delta}=\left(\vec{n}-\vec{n}^{\prime}\right) .
$$

Here $I_{0}$ is an incident beam intensity, $f(\vec{n}-\vec{n})$ is the detector instrumental function assumed to be a Gaussian, and the unit vector $\vec{n}^{\prime}$ defines the direction of the wave vector $\vec{p}$. Because of in reflectometry experiments detectors with small angular resolution $\left(\delta_{x} \sim \delta_{y} \ll 1\right)$ are used, the integration over $\vec{n}^{\prime}$ in Eq. (33) is carried out in the plane perpendicular to $\vec{n}$. The differential cross section (27) is related, however, to the sample surface, ${ }^{1}$ and therefore the variations of the wave vector and the vector $\vec{\Delta}$ are connected as

$$
q_{x}=k_{z} \Delta_{x}, \quad q_{y}=k \Delta_{y} .
$$

Substituting (27) into (33), the number of photons scattered by the incident at angle $\alpha$ to the sample surface beam and detected in the direction of specular reflection is

$$
\begin{aligned}
& N(\alpha)=N_{0} \frac{k^{4}|\chi|^{2}}{4 k_{z}^{2}}\left\{\left|T^{(0)}\left(k_{z},\left\{\Lambda^{(1)}(z)\right\}\right)+\xi B\left(k_{z}\right)\right|^{2}\right. \\
& +2 \xi^{2} \operatorname{Re}\left[T^{(0) *}\left(k_{z},\left\{\Lambda^{(1)}(z)\right\}\right) T^{(2)}\left(k_{z}\right)\right]
\end{aligned}
$$

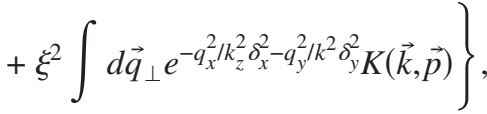

where $N_{0}=I_{0} S k_{z} / k$ is a number of photons at the sample 


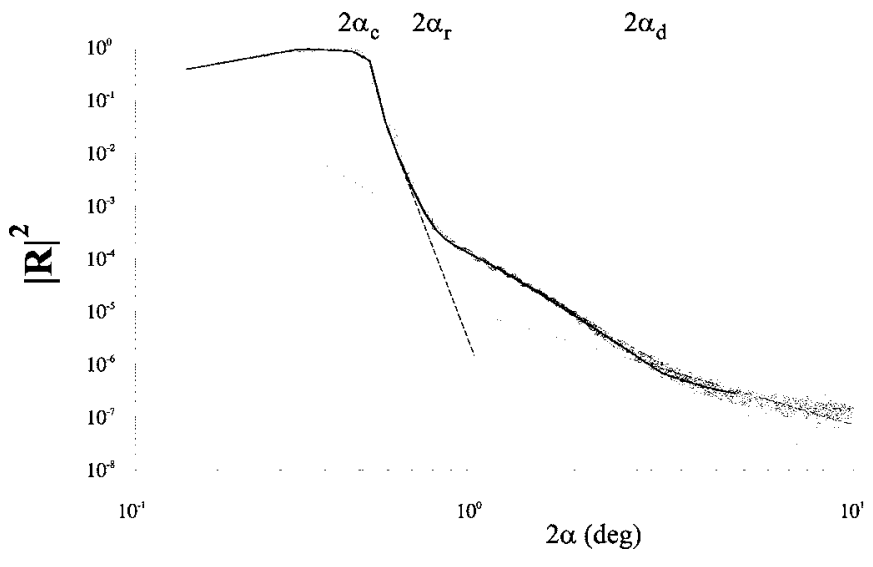

FIG. 3. The fitted by SCA experimental reflectivity from a $\mathrm{Si}_{0.65} \mathrm{Ge}_{0.35}$ sample with large roughness (Ref. 6). For the fitting of the entire area of scattering angle, formula (45) has been used with the following parameters: $\sigma=45 \AA, h=2$, and $\nu=k^{2} \alpha_{c} \delta_{x} \delta_{y} L_{c}^{2}$ $=0.012$. For comparison the dashed line shows the reflected intensity renormalized by conventional Nevot-Croce factor. The vertical lines distinguish the angle areas with different behavior of x-ray reflectivity.

surface in time unit, and $\vec{p}_{\perp}=\vec{k}_{\perp}+\vec{q}_{\perp}$ and $p_{z}=\sqrt{k^{2}-p_{\perp}^{2}}$.

For any profile $\Lambda^{(1)}(z)$, the main contribution to Eq. (35) in the region of small scattering angles is given by the term $\sim\left|T^{(0)}\left(k_{z},\left\{\Lambda^{(1)}(z)\right\}\right)\right|^{2}$. However, for scattering angles larger than total external reflection angle $\alpha>\alpha_{c}=\sqrt{\left|\chi_{0}\right|}$, the specular reflection coefficient decreases drastically, and correlation effects, being included in Eq. (35), become essential. The profile $\Lambda^{(1)}(z)$ has to be selected in a way that the sum of the following terms is equal to zero for all $k_{z}$ :

$$
\begin{aligned}
2 \operatorname{Re}\left\{T^{(0)^{*}}\left(k_{z},\left\{\Lambda^{(1)}(z)\right\}\right)\left[\xi B\left(k_{z}\right)+\xi^{2} T^{(2)}\left(k_{z}\right)\right]\right\}+\xi^{2}\left|B\left(k_{z}\right)\right|^{2} \\
\quad+\xi^{2} \int d \vec{q}_{\perp} e^{-q_{x}^{2} / k_{z}^{2} \delta_{x}^{2}-q_{y}^{2} / k^{2} \delta_{y}^{2} K(\vec{k}, \vec{p})=0 .}
\end{aligned}
$$

Then the number of detected photons (35) is

$$
N(\alpha)=N_{0}\left|R_{s}^{(0)}\left(k_{z},\left\{\Lambda^{(1)}(z)\right\}\right)\right|^{2},
$$

where the specular reflectivity $R_{s}^{(0)}$ is calculated from Eq. (20) and depends on the amplitude of coherent scattering (28), according to ${ }^{7}$

$$
\frac{k^{2} \chi}{2} T^{(0)}\left(k_{z},\left\{\Lambda^{(1)}(z)\right\}\right)=-k_{z} R_{s}^{(0)}\left(k_{z},\left\{\Lambda^{(1)}(z)\right\}\right) .
$$

To qualitatively consider the correlation effects in x-ray reflectivity, the measured ${ }^{6} \mathrm{x}$-ray reflectivity (Fig. 3) from a rough $\mathrm{Si}_{0.65} \mathrm{Ge}_{0.35}$ surface is analyzed. The x-ray polarizabil- ity of the sample is $\chi_{0}=-1.99 \times 10^{-5}+i 5.27 \times 10^{-7}$, and $\mathrm{x}$ rays with wavelength $\mathrm{Cu} K \alpha$ have been used. The scanning angle $\alpha$ is in degrees, and the intensity is normalized to unity.

The theoretical surface profile $\Lambda^{(0)}(z)$ is chosen according to Eq. (24) as an error function if the Gaussian roughness distribution and first-order DWBA are assumed. The analytical function $\varphi_{k_{z}}^{(0)}(z)$ and reflection coefficient $\mid R_{s}^{(0)}\left(k_{z}\right.$, $\left.\left\{\Lambda^{(0)}(z)\right\}\right)\left.\right|^{2}$ have also been calculated and are shown by the dashed line in Fig. 3. The fit obtained demonstrates a good agreement for the angles $\alpha_{r}>\alpha>\alpha_{c}$ and roughness $\sigma$ $\approx 45.0 \AA$. For a qualitative analysis of the reflectivity curve, an additional parameter $\alpha_{r}$ has been introduced to distinguish different regions of scanning angles. This parameter determines the range where the specular reflection from an averaged interface profile dominates over the diffuse scatteringi.e., the interval where the intensity drops exponentially:

$$
\alpha_{r} \approx \frac{1}{2 k \sigma} \text {. }
$$

For the angles $\alpha>\alpha_{r}$, however, the intensity decreases as a power function, and therefore the renormalization $\left|R_{s}^{(0)}\left(k_{z},\left\{\Lambda^{(1)}(z)\right\}\right)\right|^{2}$ due to second-order DWBA corrections is essential. To determine a profile $\Lambda^{(1)}(z)$ accounting for correlations, Eq. (36) has to be solved. As the solution for this equation is convenient to obtain directly a specular amplitude instead of the profile $\Lambda^{(1)}(z)$,

$$
T^{(0)}\left(k_{z},\left\{\Lambda^{(1)}(z)\right\}\right)=\int_{-\infty}^{\infty} d z e^{i k_{z} z} \Lambda^{(1)}(z) \varphi_{k_{z}}^{(1)}(z),
$$

where the function $\varphi_{k_{z}}^{(1)}(z)$ is a solution of the wave equation for the considered profile of the transition layer.

The solving of cumbersome integral equation is simplified due to the following reasons: (i) accuracy up to the second order of $\xi$ is required, and (ii) the angular region $\alpha>\alpha_{r}$ is considered, where the reflection coefficient is small independently of the profile shape. In this angular region,

$$
\varphi_{k_{z}}^{(1)}(z) \approx \varphi_{k_{z}}^{(0)}(z) \approx e^{i k_{z} z}
$$

and the terms of Eq. (35) are

$$
\begin{gathered}
T^{(0)}\left(k_{z},\left\{\Lambda^{(1)}(z)\right\}\right) \approx T^{(0)}\left(k_{z},\left\{\Lambda^{(0)}(z)\right\}\right)-B^{(0)}\left(k_{z}\right), \\
B\left(k_{z}\right) \approx B^{(0)}\left(k_{z}\right)=\int_{-\infty}^{\infty} d z e^{i k_{z} z} \Lambda^{(1)}(z) \varphi_{k_{z}}^{(0)}(z) .
\end{gathered}
$$

Then Eq. (35) is resolved with respect to $B^{(0)}\left(k_{z}\right)$, and the expression for the scattered amplitude is obtained assuming $\xi=1$ and with the above-mentioned accuracy:

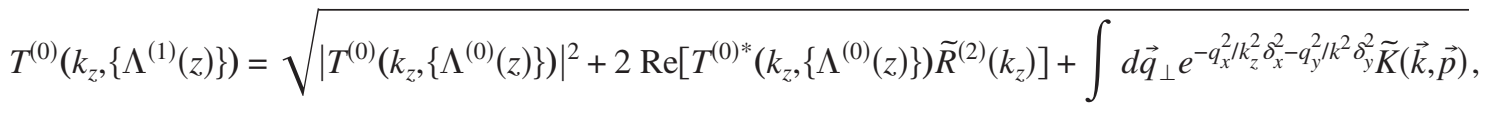

where $\widetilde{R}^{(2)}$ and $\widetilde{K}$ correspond to profile $\Lambda^{(0)}(z)$. The correction $\Lambda^{(1)}(z)$ for zeroth-order profile is calculated using Fourier transformation of (41), and generally it depends on both $z$ and $k_{z}$. 
Similarly to Ref. 5, the approximation (40) for function $\varphi_{k_{z}}^{(0)}(z)$ is used for the calculation of the amplitudes (42). Moreover, the influence of the amplitude $\widetilde{R}^{(2)}$ is negligible at the considered angles because of exponentially decreasing $T^{(0) *}\left(k_{z}\right)$. Averaging $\widetilde{K}(\vec{k}, \vec{p})$ over the roughness distribution with the function (26), the following expression for the $\mathrm{x}$-ray intensity in the direction of the specular beam is

$$
N(\alpha)=N_{0}\left\{\left|R_{s}^{(0)}(\alpha)\right|^{2}+\frac{k^{5}|\chi|^{2}}{4 \pi^{2} k_{z}^{3}} \pi \delta_{x} \delta_{y} \int_{-\infty}^{\infty} d X \int_{-\infty}^{\infty} d Y e^{-k_{z}^{2} \delta_{x}^{2} X^{2} / 4-k^{2} \delta_{y}^{2} Y^{2} / 4}\left[e^{-k_{z}^{2} \sigma^{2}\left[1-g\left(R_{\perp}\right)\right]}-e^{\left.-k_{z}^{2} \sigma^{2}\right]}\right\} .\right.
$$

For the angles $\alpha>\alpha_{r}$, the dependence of the specularly reflected intensity on the incidence angle is mostly governed by the second term in Eq. (43) and decreases as a power function:

$$
N(\alpha) \sim N_{0} \frac{1}{\alpha^{4}}
$$

The tiny oscillations within the area between $1^{\circ}$ and $2^{\circ}$ of the exit angle, which are not explained by presented approach, may be associated with the special sample structure. The large roughness of the surface has been artificially created by large-scale gratings on the surface, which are supposed to be random. However, some ordering of this grating is probable, which causes the oscillating behavior of the reflectivity curve. Another effect influencing the behavior of the curve is seen in the region $\alpha<\alpha_{c}$, where the decrease of the reflection coefficient occurs. ${ }^{2}$ This effect is due to an effective reduction of sample size $L_{S}$ at small scattering angles comparatively to that illuminated by an x-ray beam of size $L_{B}$ and area $L_{B} / \sin \alpha$. This effect is taken into account by modification of Eq. (43) with Heaviside function ${ }^{2}$

$$
N_{\mathrm{eff}}(\alpha)=N(\alpha) H\left(\sin \alpha-\frac{L_{B}}{L_{S}}\right)+\sin \alpha N(\alpha) H\left(\frac{L_{B}}{L_{S}}-\sin \alpha\right) .
$$

Formulas (43) and (45) have been used to fit the experimental data in Fig. 3. For convenience reasons, the vertical lines separate the regions of qualitatively different behavior of the reflectivity curve. The best fit is found for $\sigma=45 \AA, h=2$. An additional parameter has been introduced regulating the contribution of diffuse scattering [second term in Eq. (43)] into the total intensity:

$$
\nu=k^{2} \alpha_{c} \delta_{x} \delta_{y} L_{c}^{2}=\frac{L_{c}^{2}}{S_{c o h}} .
$$

The physical meaning of this parameter is the ratio of the area illuminated by the $\mathrm{x}$-ray sample, where the correlations are essential, to the tangential coherence area $S_{c o h}$, which is set up by angular resolution of the detector. ${ }^{2}$ The fitting in Fig. 3 is obtained at $\nu_{\text {opt }} \approx 0.012$. The specular x-ray reflectivity depends on the correlation length through the dimensionless parameter (46), which also contains the detector parameters $\delta_{x}, \delta_{y}$. The draft value of $L_{c}$ in the considered experiment is found to be $L_{c} \approx 700 \AA$, which follows from $\delta_{x} \approx \delta_{y} \approx 0.1 \alpha_{c}$.

For x-ray reflectometry, one more characteristic parameter has to be considered: the angle $\alpha_{d}$, after which reflectivity depends dominantly on detector noise, background, geometrical factors, measurement dynamical range, beam size, parameters $\delta_{x}, \delta_{y}$, etc. The nonexponential behavior of the reflection coefficient due to correlation effects in the intermediate angular region $\alpha_{d}>\alpha>\alpha_{r}$ is essential when the condition

$$
\alpha_{r}=\frac{1}{k \sigma}<\alpha_{d}
$$

is fulfilled. ${ }^{6}$ The value $\alpha_{d}$ depends on the dynamical range of the detector used in the experiment. Therefore, the roughness amplitude, at which the correlations can be observed in the reflection coefficient, depends also on experimental conditions

$$
\sigma>\frac{1}{k \alpha_{d}} .
$$

For smaller roughness amplitudes, the Nevot-Croce factor is satisfactory for data fitting. The upper limit for $\sigma$ is defined by the applicability of the DWBA; i.e., the spectral density of the specular (coherent) beam is higher than the one of the diffuse (incoherent) intensity: ${ }^{3}$

$$
\sigma<\frac{1}{k \alpha_{c}} .
$$

For the experimental data considered here, (49) results in $\sigma$ $<70 \AA$.

\section{CONCLUSIONS}

Further modification of the distorted-wave Born approximation for specular $\mathrm{x}$-ray scattering from rough surfaces and interfaces is considered, which uses a self-consistent method for the profile of interface transition layer. The approach reported permits one to take into account the change of profile due to roughness correlations. Using the SCA method, the reflection coefficient can be calculated for each DWBA order without any additional assumptions on its asymptotic behavior. The experimental x-ray data from rough sample surfaces are evaluated, and the behavior of the reflectivity is analyzed for various scattering angle regions. 
*Electronic address: alex.ulyanenkov@bruker-axs.de

${ }^{1}$ S. K. Sinha, E. B. Sirota, S. Garoff, and H. B. Stanley, Phys. Rev. B 38, 2297 (1988).

${ }^{2}$ U. Pietsch, V. Holy, and T. Baumbach, High-Resolution X-Ray Scattering: From Thin Films to Lateral Nanostructures (Springer-Verlag, Heidelberg, 2004).

${ }^{3}$ S. Dietrich and A. Haase, Phys. Rep. 260, 1 (1995).

${ }^{4}$ L. Nevot and P. Croce, Rev. Phys. Appl. 15, 761 (1980).

${ }^{5}$ D. K. G. de Boer, Phys. Rev. B 49, 5817 (1994); 51, 5297 (1995); 53, 6048 (1996).

${ }^{6}$ I. D. Feranchuk, A. A. Minkevich, and A. P. Ulyanenkov, Eur. Phys. J.: Appl. Phys. 24, 21 (2003).
${ }^{7}$ I. D. Feranchuk, S. I. Feranchuk, L. I. Komarov, S. Sytova, and A. Ulyanenkov, Phys. Rev. B 67, 235417 (2003).

${ }^{8}$ I. D. Feranchuk, L. I. Komarov, I. V. Nichipor, and A. P. Ulyanenkov, Ann. Phys. (N.Y.) 238, 370 (1995).

${ }^{9}$ T. A. Leskova and A. A. Maradudin, Waves Random Media 7, 398 (1997).

${ }^{10}$ A. V. Andreev, Phys. Lett. A 219, 349 (1996).

${ }^{11}$ W. Weber and B. Lengeler, Phys. Rev. B 46, 7953 (1992).

${ }^{12}$ S. S. Fanchenko and A. A. Nefedov, Phys. Status Solidi B 212, R3 (1999).

${ }^{13}$ A. Caticha, Phys. Rev. B 52, 9214 (1995).

${ }^{14}$ L. G. Parratt, Phys. Rev. 95, 359 (1954). 